\title{
Pharmacodynamics of TRPV1 Agonists in a Bioassay Using Human PC-3 Cells
}

\author{
Daniel Alvarez-Berdugo, ${ }^{1}$ Marcel Jiménez, ${ }^{2,3}$ Pere Clavé, ${ }^{1,2}$ and Laia Rofes ${ }^{2}$ \\ ${ }^{1}$ Laboratori de Fisiologia Digestiva, Departament de Cirurgia, Hospital de Mataró, Universitat Autònoma de Barcelona, \\ 08304 Mataró, Spain \\ ${ }^{2}$ Centro de Investigación Biomédica en Red de Enfermedades Hepáticas y Digestivas, 28029 Madrid, Spain \\ ${ }^{3}$ Departament de Biologia Cel.lular, Fisiologia i Immunologia, Universitat Autònoma de Barcelona (UAB), 08193 Bellaterra, Spain
}

Correspondence should be addressed to Pere Clavé; pere.clave@ciberehd.org

Received 19 August 2013; Accepted 5 December 2013; Published 2 February 2014

Academic Editors: J.-T. Cheng and E. Hopper-Borge

Copyright (C) 2014 Daniel Alvarez-Berdugo et al. This is an open access article distributed under the Creative Commons Attribution License, which permits unrestricted use, distribution, and reproduction in any medium, provided the original work is properly cited.

Purpose. TRPV1 is a multimodal channel mainly expressed in sensory neurons. We aimed to explore the pharmacodynamics of the TRPV1 agonists, capsaicin, natural capsaicinoids, and piperine in an in vitro bioassay using human PC-3 cells and to examine desensitization and the effect of the specific antagonist SB366791. Methods. PC-3 cells expressing TRPV1 were incubated with Fluo-4. Fluorescence emission changes following exposition to agonists with and without preincubation with antagonists were assessed and referred to maximal fluorescence following the addition of ionomycin. Concentration-response curves were fitted to the Hill equation. Results. Capsaicin and piperine had similar pharmacodynamics ( $E_{\max } 204.8 \pm 184.3 \%$ piperine versus $176.6 \pm 35.83 \%$ capsaicin, $P=0.8814$, Hill coefficient $0.70 \pm 0.50$ piperine versus $1.59 \pm 0.86$ capsaicin, $P=0.3752)$. In contrast, capsaicinoids had lower $E_{\max }(40.99 \pm 6.14 \%$ capsaicinoids versus $176.6 \pm 35.83 \%$ capsaicin, $P<0.001)$. All the TRPV1 agonists showed significant desensitization after the second exposition and their effects were strongly inhibited by SB366791. Conclusion. TRPV1 receptor is successfully stimulated by capsaicin, piperine, and natural capsaicinoids. These agonists present desensitization and their effect is significantly reduced by a TRPV1-specific antagonist. In addition, PC-3 cell bioassays proved useful in the study of TRPV1 pharmacodynamics.

\section{Introduction}

The transient receptor potential family $\mathrm{V}$ member 1 cation channel (TRPV1) is a nonselective channel that responds to noxious stimuli such as low $\mathrm{pH}$, painful heat, and irritants. Identification of TRPV1 through molecular cloning such as the capsaicin receptor [1] represented a milestone in the study of noxious stimuli. Since then, many studies have tried to determine its druggability and pharmacological characterization [2-4].

TRPV1 is mainly expressed in unmyelinated C fibers and in $A \delta$ thinly myelinated fibers of the dorsal root (DRG), trigeminal ganglion (TG), and visceral afferent fibers and it plays an important role in temperature and pain perception. However, TRPV1 expression is not exclusive to neuronal tissue but can be found in other locations such as urothelial and epithelial cells $[5,6]$, which respond first to irritating and inflammatory stimuli together with afferent nociceptors $[7,8]$.

Upregulation of TRPV1 expression has been observed in various diseases [9] including those associated with visceral hypersensitivity such as irritable bowel syndrome [10] and other diseases like chronic cough [11] and neuropathic pain [12]. Downregulation of TRPV1 has been observed in some diseases such as periodontitis [13]. Strategies aiming to block or desensitize TRPV1 have been explored to treat several diseases such as neuropathic pain, spinal detrusor hyperreflexia, bladder hypersensitivity, and pruritus [3]. Hence, TRPV1 agonists and antagonists have become essential pharmacological tools to address the treatment of these disorders.

Most bioassays aiming to characterize the pharmacodynamics of these TRPV1 ligands use human cells such as HEK293 heterologously expressing TRPV1 [14]. However, this technology is not available for most research groups 
and has some limitations in studies concerning excitable receptors due to the differences between the original cell and the engineered one [15]. There are also problems derived from the heterologous expression technique such as insertional alterations. Recently, Sánchez et al. [16] demonstrated the natural expression of TRPV1 in two human prostate cell cultures: PC-3 and LNCaP. They did this by means of retrotranscription polymerase chain reaction (RT-PCR), Western Blot, and binding studies. Thus, PC-3 was shown to be a human prostate epithelial cancer cell line that could be used to examine TRPV family receptors pharmacodynamics in future studies.

The aim of the present study was to explore the pharmacodynamics of TRPV1 agonists (capsaicin, natural capsaicinoids, and piperine) in a bioassay using human PC-3 cells prior to performing a clinical study.

\section{Material and Methods}

2.1. Experimental Design. In this bioassay, we compared the pharmacodynamics of capsaicin, natural capsaicinoids, and piperine and studied the effect of desensitization after repeated exposure and the effect of the specific TRPV1 antagonist, SB366791.

2.2. Cell Culture and Calcium Imaging. Human Caucasian prostate adenocarcinoma PC-3 cells expressing TRPV1 (ECACC Catalogue no. 90112714, Salisbury, UK) were grown in RPMI 1640 medium supplemented with $10 \%$ fetal bovine serum, $100 \mathrm{UI} / \mathrm{mL}$ penicillin $\mathrm{G}, 100 \mu \mathrm{g} / \mathrm{mL}$ streptomycin, and $0.25 \mu \mathrm{g} / \mathrm{mL}$ amphotericin B (all from Invitrogen, Paisley, $\mathrm{UK})$ at $37^{\circ} \mathrm{C}$ and $5 \% \mathrm{CO}_{2}$. Cells were passed every 3-4 days and only cells from passage 25 or lower were used in the study. Twenty-four hours before the experiment, cells were passed to a $35 \mathrm{~mm}$ plate with a glass cover slip. Before the assay, cells were incubated with extracellular medium (140 mM NaCl, $4.8 \mathrm{mM} \mathrm{KCl}, 1 \mathrm{mM} \mathrm{MgCl}_{2} \cdot 6 \mathrm{H}_{2} \mathrm{O}, 1.8 \mathrm{mM}$ $\mathrm{CaCl}_{2} \cdot 2 \mathrm{H}_{2} \mathrm{O}, 10 \mathrm{mM}$ glucose, and $10 \mathrm{mM}$ HEPES; $\left.\mathrm{pH} 7\right)$ containing $3.64 \mu \mathrm{M}$ of Fluo-4 AM (Molecular Probes, Eugene, OR, USA) at room temperature for $45 \mathrm{~min}$. Cover slips were then mounted in an imaging chamber and continuously perfused with the extracellular medium. The cells were imaged with IX-FLA equipment (Olympus Biosystems, Heidelberg, Germany) connected to an Olympus IX70 microscope with a $20 \mathrm{x}$ lens. The timing of the experiments consisted of $10 \mathrm{~s}$ agonist expositions, $5 \mathrm{~min}$ antagonist incubations, and $10 \mathrm{~min}$ of interexposition periods. Ionomycin $(10 \mu \mathrm{M})$ was added at the end of each experiment as a control of fluorescence. Images were recorded for $40 \mathrm{~s}$ at $2.5 \mathrm{~Hz}$, and fluorescence emission changes were measured after each agonist addition at $488 \mathrm{~nm}$.

2.3. Drugs and Reagents. Capsaicin, SB366791 (both from Tocris, Bristol, UK), piperine, and ionomycin (from Sigma Aldrich, St Louis, MO, USA) were dissolved in dimethyl sulfoxide (DMSO) $1 \mathrm{mM}$. Final concentrations were obtained by dissolution of drugs in extracellular medium. Capsaicinoid concentration in capsaicinoid sauce (McIlhenny Co, Avery Island, LA, USA) was determined using liquid chromatography (AOAC 995.03) and was $185.5 \mu \mathrm{g} / \mathrm{g}$, the final desired concentration being obtained by dissolution in extracellular medium.

2.4. Data Analysis. The analysis was performed with the Cell software (Olympus Biosystems, Heidelberg, Germany). Fluorescence from individual cells was monitored as a function of time by drawing a region surrounding the inner part of the cell membrane, the region of interest (ROI), and measuring the total fluorescent signal from this region. A total of 10 ROI were selected from each plate. Maximum intensity of fluorescence $\left(F_{\max }\right)$ was measured at the end of the experiment following the application of the $\mathrm{Ca}^{2+}$ ionophore, ionomycin $(1 \mu \mathrm{M})$. Background fluorescence was determined by measuring the fluorescent signal of a ROI without a cell and was subtracted from the increases in cell fluorescence $(\Delta F)$. These were calculated as the mean of the peak fluorescence value and the fluorescence value of 2 frames before and 2 frames after the peak, minus the basal fluorescence at the onset of the recording (the mean fluorescence value over the 10 first frames) (Figure 1). All experiments were carried out at room temperature $\left(18-20^{\circ} \mathrm{C}\right)$.

2.5. Statistical Methods. Concentration-response curves were fitted to the Hill equation. Variables in the equation are shown as mean \pm SEM and contrasted by means of a Student $t$ test for nonpaired data. The nonparametric Mann-Whitney $U$ test was used to analyze desensitization and antagonism. Statistical significance was accepted if $P$ values were less than 0.05. Statistical analysis was performed using GraphPad Prism 5.01 (San Diego, CA, USA).

\section{Results}

3.1. Pharmacodynamics of TRPV1 Agonists. Capsaicin, the reference agonist of TRPV1, and piperine caused a concentration-dependent response in PC-3 cells, achieving maximal effect at $10^{-5}$ and $10^{-3} \mathrm{M}$, respectively, with similar $E_{\max }$ $(204.8 \pm 184.3 \%$ piperine versus $176.6 \pm 35.83 \%$ capsaicin, $P=$ $0.8814)$ and Hill coefficient $(0.70 \pm 0.50$ piperine versus $1.59 \pm$ 0.86 capsaicin, $P=0.3752$ ) and not significantly different $\mathrm{EC}_{50}\left(4.14 \cdot 10^{-4} \mathrm{M}\right.$ piperine versus $1.90 \cdot 10^{-6} \mathrm{M}$ capsaicin, $P=$ $0.0675)$. Natural capsaicinoids also caused a concentrationdependent intracellular calcium increase, with significantly lower $E_{\max }$ than capsaicin $(40.99 \pm 6.14 \%$ capsaicinoids versus $176.6 \pm 35.83 \%$ capsaicin, $P<0.001)$, and similar $\mathrm{EC}_{50}$ $\left(4.36 \cdot 10^{-6} \mathrm{M}\right.$ capsaicinoids versus $1.90 \cdot 10^{-6} \mathrm{M}$ capsaicin, $P=0.1601)$ and Hill coefficient $(1.18 \pm 0.45$ capsaicinoids versus $1.59 \pm 0.86$ capsaicin, $P=0.674$ ) (Figure 2 ).

3.2. Specificity: Desensitization and the Effect of an Antagonist. Repetitive expositions to capsaicin $\left(10^{-6} \mathrm{M}, 10 \mathrm{~min}\right.$ interexposition), piperine $\left(10^{-3} \mathrm{M}\right.$ and $10^{-4} \mathrm{M}, 10 \mathrm{~min}$ interexposition), or natural capsaicinoids $1.2 \cdot 10^{-5} \mathrm{M}$ (10 min interexposition) significantly reduced the response of PC-3 cells. The effect of capsaicin was reduced by $38.31 \pm 4.08 \%$, piperine by $67.61 \pm$ $5.31 \%$, and capsaicinoids by $22.30 \pm 2.24 \%$ after second exposition (Figure 3). In addition, $5 \mathrm{~min}$ incubation with the specific TRPV1 antagonist SB366791 $\left(10^{-5} \mathrm{M}\right)$, strongly 


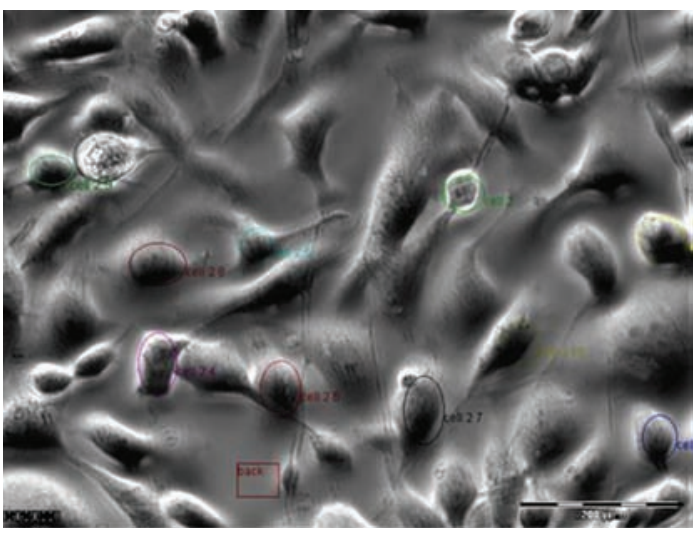

(a)

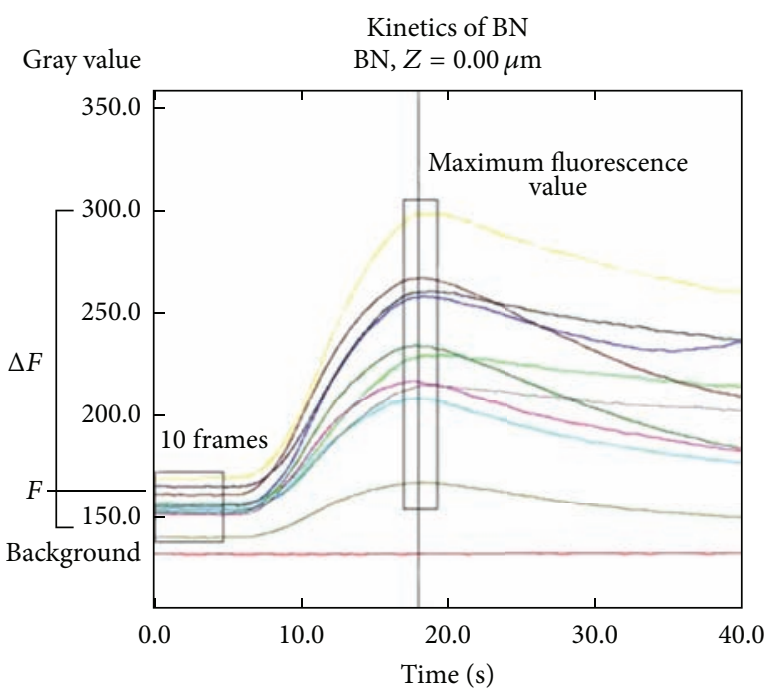

(b)

FIGURE 1: Calcium imaging data analysis. The regions of interest (ROIs) of 10 cells per plate (colored ellipsis) are marked to measure changes in fluorescence and a ROI without cells (dark red rectangle) is also marked to measure the background signal (a). Increases in fluorescence are plotted as a time function for each cell. The increase of fluorescence $(\Delta F)$ is calculated as the difference between the mean of the peak and 2 frames backward and forward (narrow rectangle measurements) and the mean of the measurements of 10 frames before agonist exposition (wide rectangle of measurements); the background signal (red line) is then subtracted (b).

antagonized the response of capsaicin $\left(10^{-6} \mathrm{M}\right)$, piperine $\left(10^{-3} \mathrm{M}\right)$, and capsaicinoids $\left(1.2 \cdot 10^{-5} \mathrm{M}\right)$. Following $5 \mathrm{~min}$ incubation with the antagonist and $5 \mathrm{~min}$ wash before second exposition, the effect of capsaicin was reduced by $74.66 \pm$ $2.93 \%$; piperine by $100 \pm 0.005 \%$, and capsaicinoids by $91.71 \pm$ $2.76 \%$ (Figure 3 ).

\section{Discussion}

The results show that capsaicin and piperine to a greater degree and natural capsaicinoids to a lesser degree successfully stimulate the TRPV1 channel. Repeated exposition of these agonists decreases the effect on TRPV1, suggesting desensitization. Moreover, their effect is significantly reduced by a TRPV1-specific antagonist, showing their action to be specific to this receptor. PC-3 cells were found to be perfectly adequate tools to study TRPV1 pharmacodynamics.

Caterina et al. studied the pharmacology of rVR1 when they first cloned it [1]. Since then, several groups have evaluated the use of TRPV1 as a therapeutic target to treat various diseases $[3,4]$. In our research, TRPV1 is a promising target to treat oropharyngeal dysphagia, a major complaint among the elderly and patients with neurological diseases, and one characterized by pharyngeal and laryngeal sensory deficits and delayed and prolonged swallow response [17]. Previous clinical studies showed capsaicin and piperine to be effective in improving the swallowing response $[18,19]$ but we needed more knowledge of the TRPV1 agonists' pharmacodynamics to design a clinical trial to assess proof of concept [20]. We used a bioassay to evaluate the pharmacology of capsaicin, piperine, and natural capsaicinoids on human TRPV1 constitutively expressed in PC-3 cells.
In our study, capsaicin and piperine had similar $E_{\max }$ and Hill coefficient values and we did not find significant differences in their $\mathrm{EC}_{50}$ values, while comparable assays found a lower maximum effect for piperine and significant differences in their $\mathrm{EC}_{50}[21,22]$. We also found that natural capsaicinoid sauce has lower $E_{\max }$ than capsaicin or piperine. This could be explained by the fact that capsaicinoid sauce contains different capsaicinoids, one of which is capsaicin, found in previous studies to have the greatest pungent effect among vanilloids [23]. Our results helped us determine the following optimal concentrations for the clinical trial: $10 \mu \mathrm{M}$ for capsaicin, $150 \mu \mathrm{M}$ to $1 \mathrm{mM}$ for piperine, and $150 \mu \mathrm{M}$ for capsaicinoid sauce [20].

Our results also showed that all the agonists tested undergo desensitization after repeated exposition. Capsaicin had already been shown to desensitize TRPV1 action [1, 21, 24], and Liu and Simon had shown that piperine also desensitizes TRPV1 action [21], but in our assay piperine desensitized TRPV1 action to a greater degree.

Finally, the use of TRPV1-specific antagonist SB-366791 allowed us to verify that the effect of our agonists is specific to TRPV1. In previous studies, capsazepine was used as a specific vanilloid receptor antagonist to assess agonist specificity, but it has nonselective actions on other receptors and apparent modality-specific properties. SB-366791, however, is a TRPV1 antagonist with high potency and improved selectivity profile with respect to other commonly used TRPV1 antagonists [25]. Another difference between our study and previous ones is the assay design with PC-3 cells, a system that has been rarely used in comparison with transfected HEK293 cells. This study shows that PC-3 cells are a good surrogate to test TRPV1 pharmacology and more accessible as they do not require transfection. 


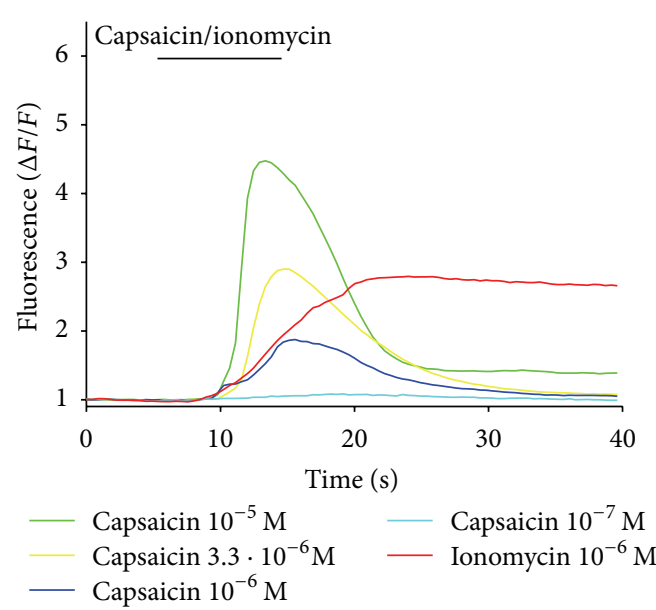

(a)

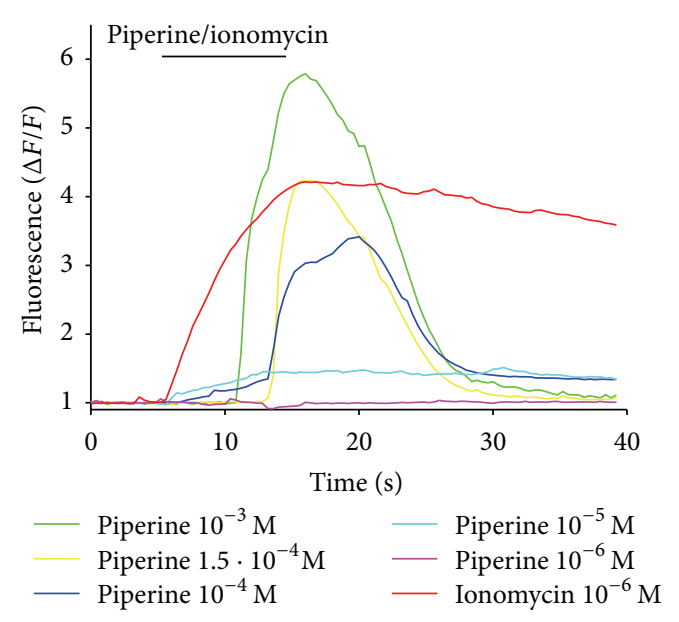

(c)

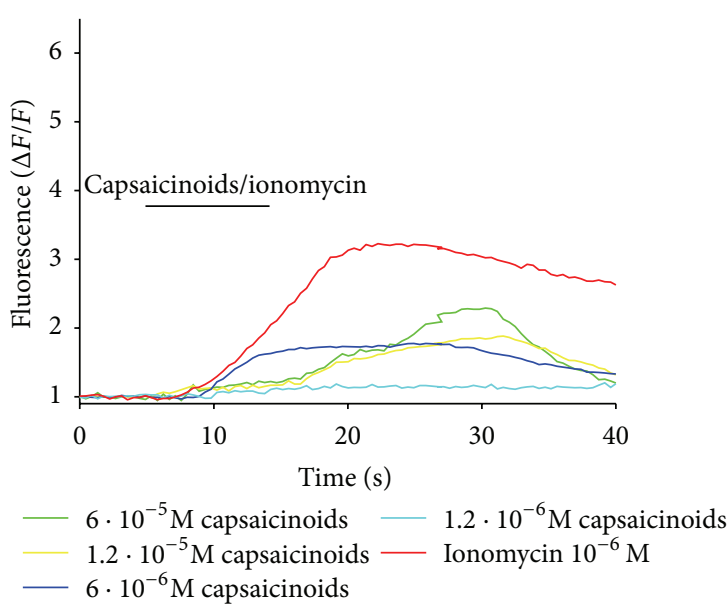

(e)

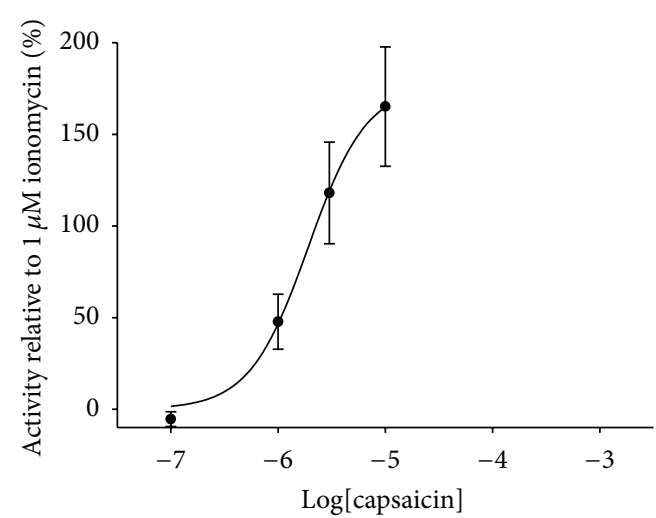

(b)

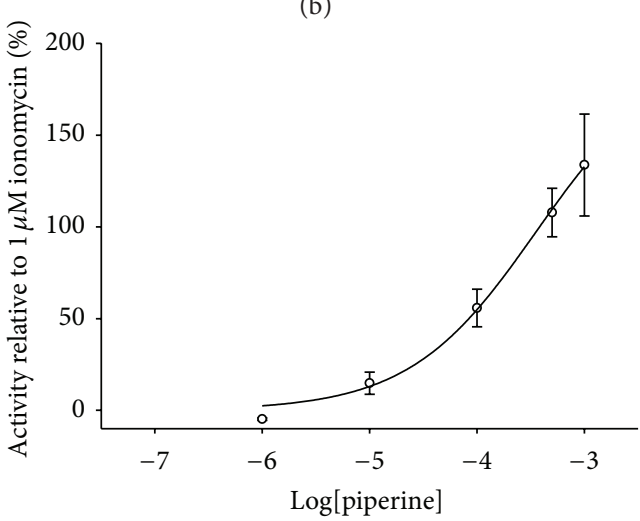

(d)

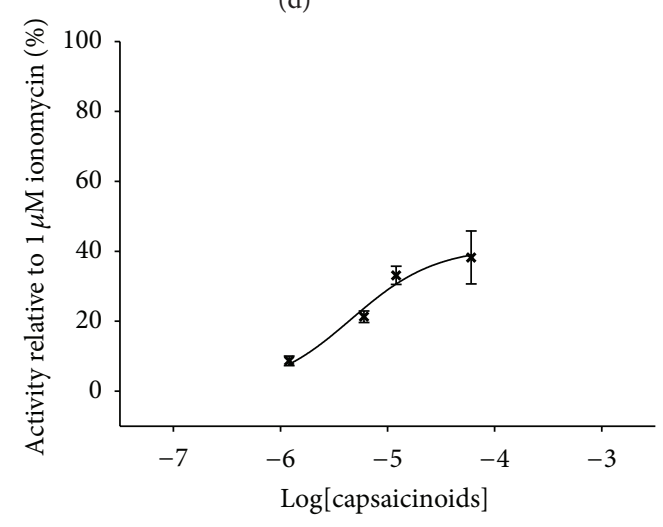

(f)

Figure 2: Calcium transients and dose-response curves. The means of measurements for each agonist concentration are plotted as time functions of the increase in fluorescence related to the signal before exposition (a, c, and e); ionomycin was applied at the end of each experiment and its maximum effect was used to normalize the effect of the different agonists and concentrations. The slope showing calcium entrance induced by capsaicin and piperine exposition (capsaicin $830.1 \pm 128.1$ relative increase of fluorescence per second and piperine $1238 \pm$ 146.8 relative increase of fluorescence per second) (a, c) is sharper than the slope showing calcium entrance induced by natural capsaicinoids exposition (184.6 \pm 17.39 relative increase of fluorescence per second) (e). The normalized effect of each concentration is plotted as a doseresponse curve for each agonist. Each point represents the mean \pm SEM of 3 independent experiments $(n=10)(b, d$, and f). 

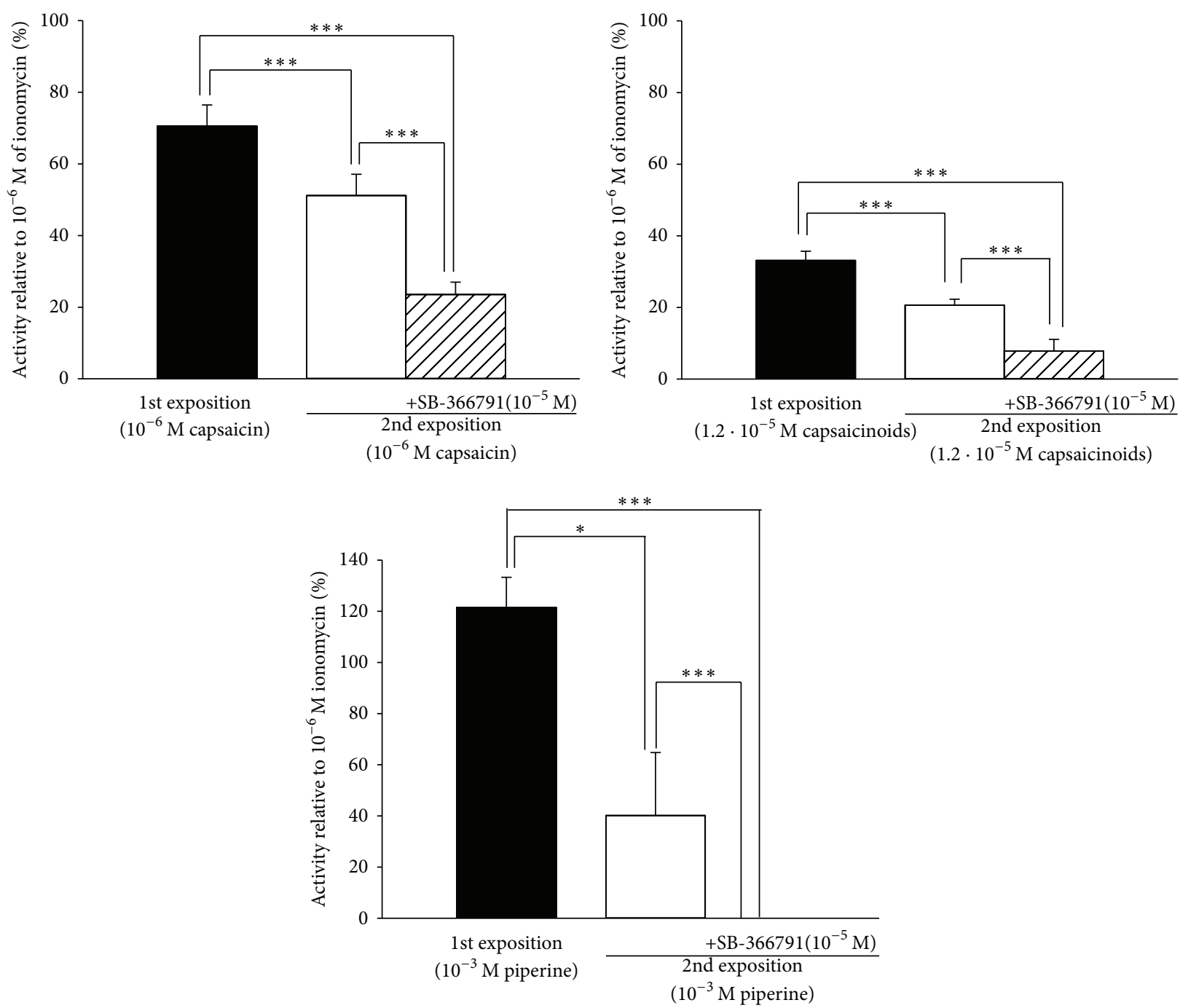

FIGURE 3: Desensitization and antagonist effect. Capsaicin, capsaicinoids, and piperine normalized first exposition effect (black box) is compared with their normalized second exposition effect (white box) and SB-366791 incubation before second exposition effect (striped box). Data is expressed as mean \pm SEM. ${ }^{*} P<0.05,{ }^{* * *} P<0.001$.

\section{Conclusion}

In summary, we have examined the pharmacological parameters of TRPV1 agonists and analysed desensitization and the effect of the antagonist SB366791 using an alternative methodology more easily accessible to most research groups. We reached our goal of testing capsaicin, piperine, and natural capsaicinoid pharmacodynamics for subsequent clinical assays.

\section{Conflict of Interests}

The authors declare that there is no conflict of interests regarding the publication of this paper.

\section{Acknowledgments}

This work was supported by grants from the Spanish Ministerio de Ciencia e Innovación (PS09/01012, INT 10/228), the Generalitat de Catalunya (2011 CTP 00032), the Agencia de Gestió d'Ajuts Universitaris i de Recerca (2009 SGR 708), and La Fundació Marató de TV3. The authors thank N. Olea and I. Díaz-Laviada from Universidad de Alcalá de Henares for generously supplying PC-3 cells and Bolton Cile (especially A de la Cruz) for kindly providing the capsaicinoids sauce. They would also like to thank C. Arenas and E. Martínez for their helpful collaboration in the present study and J. Lewis for writing assistance.

\section{References}

[1] M. J. Caterina, M. A. Schumacher, M. Tominaga, T. A. Rosen, J. D. Levine, and D. Julius, "The capsaicin receptor: a heatactivated ion channel in the pain pathway," Nature, vol. 389, no. 6653, pp. 816-824, 1997.

[2] A. Ferrer-Montiel, C. García-Martínez, C. Morenilla-Palao et al., "Molecular architecture of the vanilloid receptor: insights for drug design," European Journal of Biochemistry, vol. 271, no. 10, pp. 1820-1826, 2004. 
[3] A. Szallasi and P. M. Blumberg, "Vanilloid (Capsaicin) receptors and mechanisms," Pharmacological Reviews, vol. 51, no. 2, pp. 159-211, 1999.

[4] O. Sterner and A. Szallasi, "Novel natural vanilloid receptor agonists: new therapeutic targets for drug development," Trends in Pharmacological Sciences, vol. 20, no. 11, pp. 459-465, 1999.

[5] A. Avelino, C. Cruz, I. Nagy, and F. Cruz, "Vanilloid receptor 1 expression in the rat urinary tract," Neuroscience, vol. 109, no. 4, pp. 787-798, 2002.

[6] K. Inoue, S. Koizumi, S. Fuziwara, S. Denda, K. Inoue, and M. Denda, "Functional vanilloid receptors in cultured normal human epidermal keratinocytes," Biochemical and Biophysical Research Communications, vol. 291, no. 1, pp. 124-129, 2002.

[7] M. J. Caterina, "Vanilloid receptors take a TRP beyond the sensory afferent," Pain, vol. 105, no. 1-2, pp. 5-9, 2003.

[8] M. D. Southall, T. Li, L. S. Gharibova, Y. Pei, G. D. Nicol, and J. B. Travers, "Activation of epidermal vanilloid receptor-1 induces release of proinflammatory mediators in human keratinocytes," Journal of Pharmacology and Experimental Therapeutics, vol. 304, no. 1, pp. 217-222, 2003.

[9] A. Szallasi, "Vanilloid (capsaicin) receptors in health and disease," American Journal of Clinical Pathology, vol. 118, no. 1, pp. 110-121, 2002.

[10] A. Akbar, Y. Yiangou, P. Facer, J. R. F. Walters, P. Anand, and S. Ghosh, "Increased capsaicin receptor TRPV1-expressing sensory fibres in irritable bowel syndrome and their correlation with abdominal pain," Gut, vol. 57, no. 7, pp. 923-929, 2008.

[11] D. A. Groneberg, A. Niimi, Q. T. Dinh et al., "Increased expression of transient receptor potential vanilloid-1 in airway nerves of chronic cough," American Journal of Respiratory and Critical Care Medicine, vol. 170, no. 12, pp. 1276-1280, 2004.

[12] H. Urano, T. Ara, and Y. Fujinami, "Aberrant TRPV1 expression in heat hyperalgesia associated with trigeminal neuropathic pain," Journal of Pharmacological Sciences, vol. 118, pp. 690-697, 2012.

[13] A. Öztürk and L. Yildiz, "Expression of transient receptor potential vanilloid receptor 1 and toll-like receptor 4 in aggressive periodontitis and in chronic periodontitis," Journal of Periodontal Research, vol. 46, no. 4, pp. 475-482, 2011.

[14] M. Tominaga, M. J. Caterina, A. B. Malmberg et al., "The cloned capsaicin receptor integrates multiple pain-producing stimuli," Neuron, vol. 21, no. 3, pp. 531-543, 1998.

[15] H. A. Lester, "Heterologous expression of excitability proteins: route to more specific drugs?” Science, vol. 241, no. 4869, pp. 1057-1063, 1988.

[16] M. G. Sánchez, A. M. Sánchez, B. Collado et al., "Expression of the Transient Receptor Potential Vanilloid 1 (TRPV1) in LNCaP and PC-3 prostate cancer cells and in human prostate tissue," European Journal of Pharmacology, vol. 515, no. 1-3, pp. 20-27, 2005.

[17] P. Clavé, R. Terré, M. de Kraa, and M. Serra, “Approaching oropharyngeal dysphagia," Revista Espanola de Enfermedades Digestivas, vol. 96, no. 2, pp. 119-131, 2004.

[18] T. Ebihara, H. Takahashi, S. Ebihara et al., "Capsaicin troche for swallowing dysfunction in older people," Journal of the American Geriatrics Society, vol. 53, no. 5, pp. 824-828, 2005.

[19] T. Ebihara, S. Ebihara, M. Maruyama et al., "A randomized trial of olfactory stimulation using black pepper oil in older people with swallowing dysfunction," Journal of the American Geriatrics Society, vol. 54, no. 9, pp. 1401-1406, 2006.
[20] L. Rofes, V. Arreola, A. Martin, and P. Clave, "Natural capsaicinoids improve swallow response in older patients with oropharyngeal dysphagia," Gut, vol. 62, no. 9, pp. 1280-1287, 2013.

[21] L. Liu and S. A. Simon, "Similarities and differences in the currents activated by capsaicin, piperine, and zingerone in rat trigeminal ganglion cells," Journal of Neurophysiology, vol. 76, no. 3, pp. 1858-1869, 1996.

[22] Y. Okumura, M. Narukawa, Y. Iwasaki et al., "Activation of TRPV1 and TRPA1 by black pepper components," Bioscience, Biotechnology and Biochemistry, vol. 74, no. 5, pp. 1068-1072, 2010.

[23] D. Ursu, K. Knopp, R. E. Beattie, B. Liu, and E. Sher, "Pungency of TRPV1 agonists is directly correlated with kinetics of receptor activation and lipophilicity," European Journal of Pharmacology, vol. 641, no. 2-3, pp. 114-122, 2010.

[24] P. Hayes, H. J. Meadows, M. J. Gunthorpe et al., "Cloning and functional expression of a human orthologue of rat vanilloid receptor-1," Pain, vol. 88, no. 2, pp. 205-215, 2000.

[25] M. J. Gunthorpe, H. K. Rami, J. C. Jerman et al., "Identification and characterisation of SB-366791, a potent and selective vanilloid receptor (VR1/TRPV1) antagonist," Neuropharmacology, vol. 46, no. 1, pp. 133-149, 2004. 

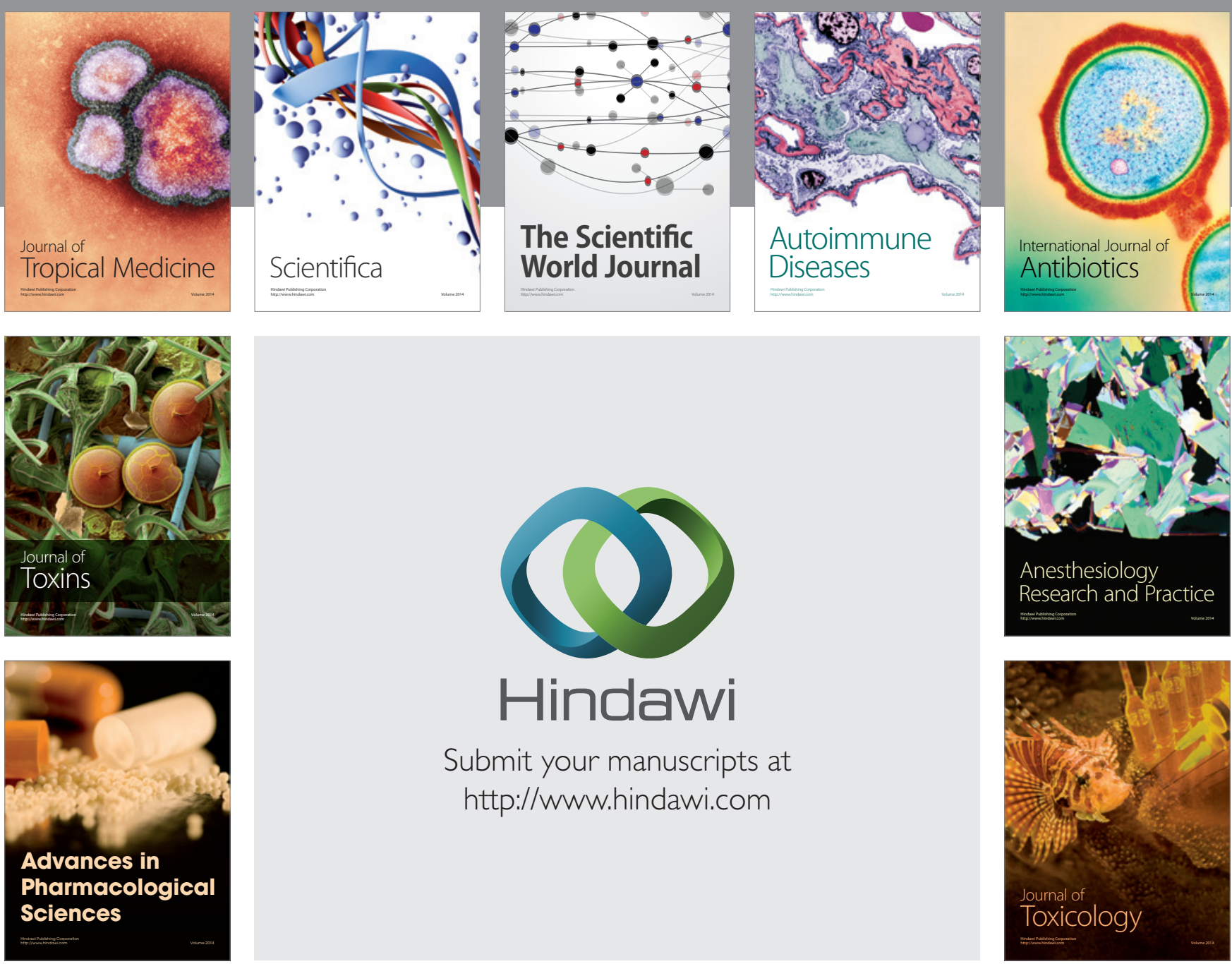

\section{Hindawi}

Submit your manuscripts at

http://www.hindawi.com
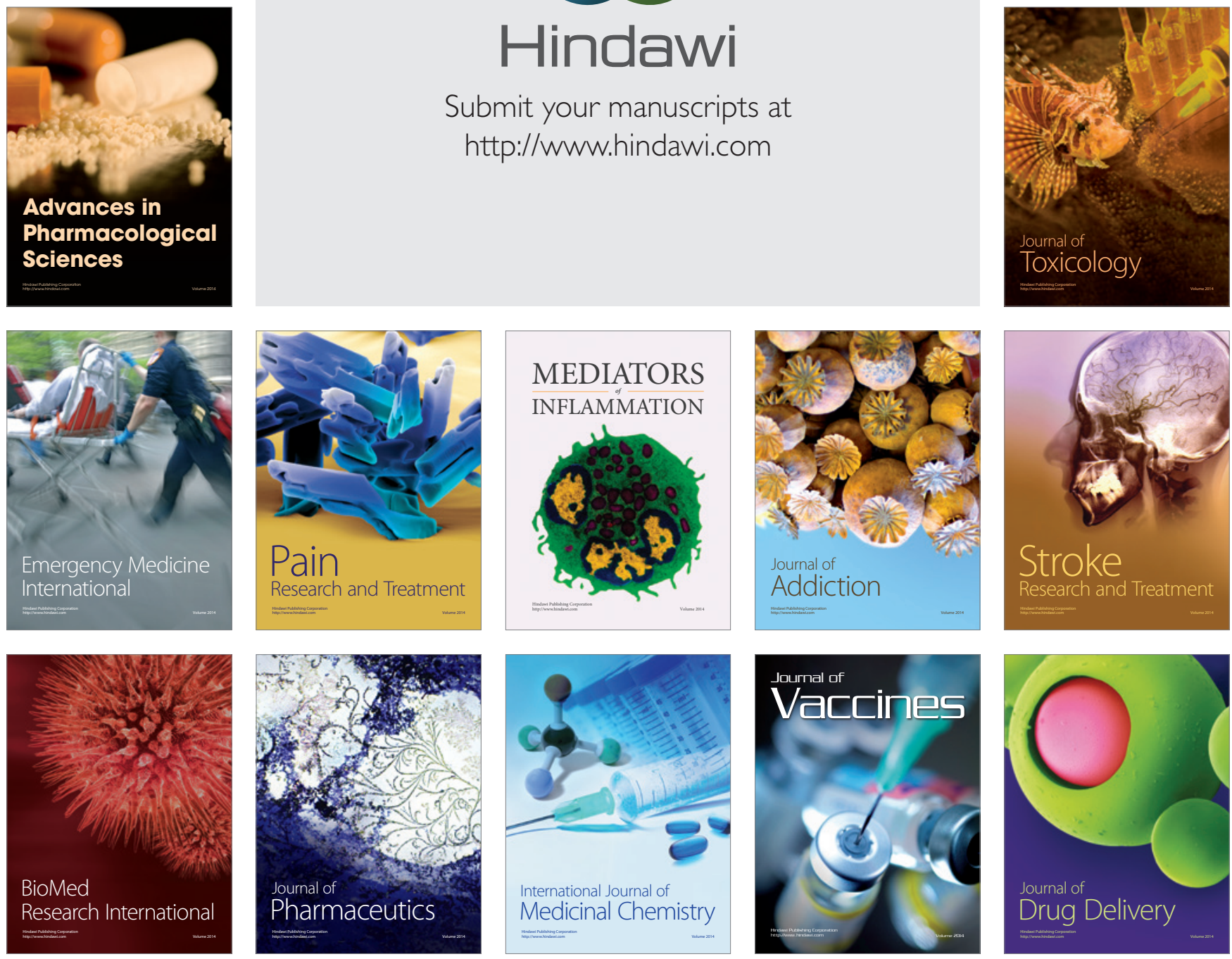